\title{
Serum Tartrate-resistant Acid Phosphatase-5b Levels are Associated with the Severity and Extent of Coronary Atherosclerosis in Patients with Coronary Artery Disease
}

\author{
Taichirou Morisawa ${ }^{1}$, Akihiro Nakagomi ${ }^{1}$, Keiichi Kohashi ${ }^{1}$, Yoshiki Kusama ${ }^{1}$ and Wataru Shimizu ${ }^{2}$ \\ ${ }^{1}$ Department of Internal Medicine and Cardiology, Tama-Nagayama Hospital, Nippon Medical School, Tokyo, Japan \\ ${ }^{2}$ Department of Cardiovascular Medicine, Nippon Medical School, Tokyo, Japan
}

\begin{abstract}
Aims: Tartrate-resistant acid phosphatase (TRACP)-5b and osteoprotegerin (OPG) are specific and sensitive markers of bone resorption in patients with rheumatoid arthritis (RA) and chronic kidney disease (CKD). The TRACP-5b level is associated with the severity of RA and CKD, while the OPG level is associated with the severity of coronary atherosclerosis and calcification, and can predict a poor outcome in patients with coronary artery disease (CAD). However, the impact of TRACP-5b on coronary atherosclerosis in CAD patients remains unclear.

Methods: A total of 71 CAD patients $(57$ men, 14 women; mean age: $69.0 \pm 9.7$ years $)$ and 28 ageand gender- matched healthy subjects were investigated. The number of diseased vessels (a marker of the severity of coronary atherosclerosis) and the Gensini score (a marker of the extent of coronary atherosclerosis), as well as the OPG and TRACP-5b levels were measured in CAD patients. The TRACP-5b levels were classified into quartiles.

Results: The TRACP-5b levels were significantly higher in CAD patients than in healthy subjects. Patients with higher TRACP-5b levels had higher OPG levels and Gensini scores than those with lower TRACP-5b levels. Higher TRACP-5b levels were associated with an increased number of diseased vessels. A multivariate linear regression analysis showed that the OPG level and the number of diseased vessels or the Gensini score were significantly and independently associated with the TRACP-5b level.

Conclusions: These data indicate that the TRACP- 5 b level is significantly associated with the OPG level and with the severity and extent of coronary atherosclerosis in CAD patients.
\end{abstract}

Key words: Tartrate-resistant acid phosphatase-5b, Osteoprotegerin, Number of diseased vessels, Gensini score, Coronary artery disease

This article is distributed under the terms of the latest version of CC BY-NC-SA defined by the Creative Commons Attribution License.

\section{Introduction}

Atherosclerosis and the calcification of the coronary arteries occur via an active process that resembles bone formation and remodeling within the vessel wall. It is controlled by complex enzymatic and cellular pathways ${ }^{1-6)}$. The tumor necrosis factor (TNF)-related family includes the receptor activator of nuclear factor $\kappa \mathrm{B}$ (RANK), RANK ligand (RANKL) and osteoprote-

Address for correspondence: Akihiro Nakagomi, 1-7-1 Nagayama, Tama City, 206-8512, Tokyo, Japan

E-mail: nakagomi@nms.ac.jp

Received: December 8, 2016

Accepted for publication: February 27, 2017 gerin $(\mathrm{OPG})$, which are involved in the regulation of vascular atherosclerosis and calcification ${ }^{2-8)}$.

OPG is a key factor in bone remodeling and is a sensitive and specific marker of bone resorption ${ }^{2)}$. Clinical studies have reported that the extent of coronary calcium reflects the total coronary atherosclerotic burden, and that OPG elevation is associated with the progression of vascular atherosclerosis and calcification in patients on long-term hemodialysis, the growth of abdominal aortic aneurysms, the presence and severity of coronary artery disease (CAD), arterial stiffness estimated by the cardio-ankle vascular index in hypertensive patients and the cardiovascular risk in the general population $^{2,9-16)}$.

Tartrate-resistant acid phosphate (TRACP), also 
known as type-5 acid phosphatase and purple acid phosphatase, is an iron-containing glycoprotein expressed in high amounts by bone-resorbing osteoclasts, inflammatory macrophages, and dendritic cells ${ }^{17)}$. Two forms of TRACP circulate in the human blood: TRACP-5a, which is derived from macrophages and dendritic cells, and TRACP-5b, which is derived from osteoclasts. It has been suggested that TRACP-5a is a biomarker of the systemic inflammatory burden in patients with chronic inflammatory diseases, including rheumatoid arthritis (RA) and sarcoidosis ${ }^{17}{ }^{18}$. Recent studies have shown that the serum TRACP-5b activity is correlated with bone resorption markers and osteoclast numbers ${ }^{17)}$. In addition, the serum TRACP-5b activity is a promising new marker with clinical relevance in diseases such as RA, chronic kidney disease (CKD), and bone metastasis in various cancers that are associated with increased bone resorption and destruction ${ }^{17,19)}$. Thus, TRACP-5b is a sensitive and specific marker of bone resorption and bone remodeling in patients with $\mathrm{RA}, \mathrm{CKD}$, and cancer with bone metastasis.

However, no studies have investigated the effects of TRACP-5b on the severity and extent of coronary atherosclerosis in CAD patients. Thus, the aim of the present study was to investigate whether or not the serum levels of TRACP-5b are associated with the severity and extent of coronary atherosclerosis in CAD patients.

\section{Methods}

\section{Study Population}

A total of 71 selected (nonconsecutive) CAD patients (57 men and 14 women; mean age: 69.0 \pm 9.7 years) were enrolled in the current study. A total of 28 age- and gender-matched normal healthy subjects $(20$ men and 8 women; mean age, $69.2 \pm 6.3$ years) were included for comparison. All of the patients and normal healthy subjects were recruited from an outpatient clinic of Tama-Nagayama Hospital, Nippon Medical School, between October 2012 and December 2013. All of the patients underwent coronary angiography (CAG) prior to their enrollment in this study.

This study included CAD patients and normal healthy subjects who were $\geq 20$ years of age. CAD was defined as stable angina with or without a history of myocardial infarction and found to have $\geq 70 \%$ stenosis in at least 1 branch of the main coronary arteries on CAG.

All patients presenting with the clinical signs of cardiogenic shock, acute decompensated heart failure, acute myocardial infarction (AMI) or unstable angina within the previous month, vasospastic angina, stroke, peripheral artery disease (PAD), or patients who had undergone percutaneous coronary intervention (PCI) or coronary artery bypass grafting (CABG) prior to enrollment, and osteoporosis patients were excluded from the present study. Patients with CAD and normal healthy subjects with clinical signs of acute infection, autoimmune disorders, severe renal (serum creatinine $>1.5 \mathrm{mg} / \mathrm{dL}$ ), hepatic disease or suspected malignancies were excluded from the study. Normal healthy subjects with osteoporosis and those with a history of cardiovascular diseases, including coronary artery disease, heart failure, cardiomyopathy and valvular heart disease, or with stroke or PAD were excluded from this study. In addition, normal healthy subjects with a history of hypertension, dyslipidemia or diabetes who had been receiving these medications before enrollment were also excluded from the present study.

Eleven patients had a history of non-ST elevation myocardial infarction and did not undergo CAG or PCI in the acute phase because they had been admitted to other hospitals more than $12 \mathrm{~h}$ after the onset of AMI without chest pain. They were referred from these hospitals for further examinations, including CAG and noninvasive exercise tests. Therefore, we performed the exercise treadmill test or stress myocardial perfusion imaging to detect myocardial ischemia at an outpatient clinic of our hospital, where these patients were found to be positive for myocardial ischemia. They were therefore allowed to participate in this study and underwent CAG for the first time at our hospital after enrollment in the present study.

The left ventricular ejection fraction (LVEF) was measured in each patient by echocardiography using a LOGIQ 7 system (GE Healthcare, Milwaukee, WI, USA) within one week of the measurement of the biochemical markers and was analyzed by two blinded trained cardiologists.

The risk factors in the patient population were as follows: smoking, with subjects defined as active smokers if they smoked at least 1 cigarette per day for $\geq 1$ year; hypertension, defined as a systolic blood pressure (SBP) of $\geq 140 \mathrm{mmHg}$ and/or diastolic blood pressure of $\geq 90 \mathrm{mmHg}$ or treatment with antihypertensive medication; diabetes, defined as a fasting plasma glucose (FPG) level of $\geq 126 \mathrm{mg} / \mathrm{dL}$, a casual plasma glucose level of $\geq 200 \mathrm{mg} / \mathrm{dL}$ or a 2 -h plasma glucose level during a 75 -g oral glucose tolerance test of $\geq 200 \mathrm{mg} /$ $\mathrm{dL}$ or treatment with anti-diabetic medication; and dyslipidemia, defined as an overnight fasting serum total cholesterol (TC) level of $\geq 220 \mathrm{mg} / \mathrm{dL}$, a triglyceride (TG) level of $\geq 150 \mathrm{mg} / \mathrm{dL}$, a low-density lipoprotein (LDL) cholesterol level of $\geq 140 \mathrm{mg} / \mathrm{dL}$, a highdensity lipoprotein (HDL) cholesterol level of $\leq 40$ $\mathrm{mg} / \mathrm{dL}$ or treatment with lipid-moderating medications prior to enrollment in the study. The LDL cholesterol 
Table 1. The demographic and clinical characteristics of the 71 CAD patients and 28 ageand gender-matched normal control subjects.

\begin{tabular}{lccc}
\hline & CAD $(n=71)$ & Normal $(n=28)$ & $P$ value \\
\hline Age (years) & $69.0 \pm 9.7$ & $69.2 \pm 6.3$ & 0.931 \\
Gender (male, $\%)$ & $57(80.3)$ & $20(71.4)$ & 0.422 \\
Body mass index $\left(\mathrm{kg} / \mathrm{m}^{2}\right)$ & $24.7 \pm 4.0$ & $23.3 \pm 1.6$ & 0.075 \\
Systolic BP $(\mathrm{mmHg})$ & $129 \pm 19$ & $122 \pm 4$ & 0.056 \\
Heart rate $(\mathrm{beats} / \mathrm{min})$ & $68 \pm 9$ & $70 \pm 7$ & 0.117 \\
TRACP-5b (mU/dL) & $362.5(285.0,482.0)$ & $300.5(248.0,334.6)$ & 0.001 \\
hs-CRP $(\mathrm{mg} / \mathrm{L})$ & $0.76(0.28,2.61)$ & $0.27(0.18,0.29)$ & $<0.001$ \\
Total cholesterol (mg/dL) & $162 \pm 31$ & $174 \pm 12$ & 0.053 \\
LDL-cholesterol $(\mathrm{mg} / \mathrm{dL})$ & $113 \pm 33$ & $100 \pm 12$ & 0.036 \\
HDL-cholesterol $(\mathrm{mg} / \mathrm{dL})$ & $52 \pm 12$ & $55 \pm 6$ & 0.227 \\
Triglycerides $(\mathrm{mg} / \mathrm{dL})$ & $115(86,149)$ & $96(79,108)$ & 0.015 \\
FPG $(\mathrm{mg} / \mathrm{dL})$ & $122 \pm 30$ & $92 \pm 8$ & $<0.001$ \\
eGFR $\left(\mathrm{mL} / \mathrm{min} / 1.73 \mathrm{~m}^{2}\right)$ & $61.5 \pm 15.9$ & $61.5 \pm 8.2$ & 0.998 \\
\hline
\end{tabular}

The data are expressed as the mean $\pm \mathrm{SD}$ or median (interquartile range).

CAD, patients with coronary artery disease; Normal, normal healthy subjects, BP, blood pressure; TRACP, tartrate-resistant acid phosphatase; hs-CRP, high-sensitivity C-reactive protein; LDL, low-density lipoprotein; HDL, high-density lipoprotein; FPG, fasting plasma glucose; eGFR, estimated glomerular filtration rate.

level was calculated using the Friedewald formula (LDL cholesterol $=$ TC - HDL cholesterol - TG/5).

The investigation protocol was approved by the Institutional Ethics Committee of Nippon Medical School, Tama-Nagayama Hospital. All patients with CAD and normal healthy subjects provided their written informed consent prior to enrollment in the study.

\section{CAG}

Patents underwent CAG for the first time at entry. The CAG findings were estimated at entry and were based on the consensus opinion of two experienced cardiologists. Coronary angiograms were scored by two techniques, as follows:

1) The number of diseased coronary vessels (severity score), including the coronary arteries with $\geq 70 \%$ (in diameter) stenosis, with scores ranging from 0 to $3^{20)}$.

2) The Gensini score is used to evaluate the extent of coronary atherosclerosis. According to this scoring system, the angiographic severity of the lesion was rated as follows: 1 point $=0 \%-25 \%, 2$ points $=25 \%-$ $50 \%, 4$ points $=50 \%-75 \%, 8$ points $=75 \%-90 \%, 16$ points $=90 \%-99 \%$ and 32 points $=100 \%$ (a completely occluded vessel). The score was multiplied by the segment location multiplying factor (left main coronary artery $=5$ points, proximal left descending branch and left circumflex artery lesion $=2.5$ points, middle left ending artery lesion $=1.5$ points, first diagonal branch and the obtuse marginal branches and right coronary artery lesion $=1$ point, and second diag- onal and left circumflex artery posterolateral branch lesion $=0.5$ points), and the results were then summed to obtain the Gensini score for each patient ${ }^{21)}$.

\section{Laboratory Measurements}

At entry, the plasma levels of OPG were measured with a specific enzyme-linked immunosorbent assay (ELISA) using a commercially available system (R\&D Systems). The intra- and inter-assay coefficients of variation were $<5 \%$ for all of the ELISAs. The serum levels of TRACP-5b were measured using a fragment-absorbed immune-capture enzyme assay (SRL Inc., Tokyo, Japan), and the intra- and interassay coefficients of variation were also $<5 \%$ for all of the assays.

The high-sensitivity C-reactive protein (hsCRP) levels were measured using an immunoturbidimetry assay. The estimated glomerular filtration rate (eGFR) was calculated according to the following equation for Japanese subjects, as recommended by the Japanese Society of Nephology: eGFR $\left(\mathrm{mL} / \mathrm{min} / 1.73 \mathrm{~m}^{2}\right)=194$ $\times$ serum creatinine ${ }^{-1.094}(\mathrm{mg} / \mathrm{dL}) \times$ age $^{-0.287}$ (years $) \times$ 0.739 , if female ${ }^{22)}$. CKD was defined as eGFR $<60$ $\mathrm{mL} / \mathrm{min} / 1.73 \mathrm{~m}^{2}$. The serum levels of albumin, TC, TG, HDL cholesterol, glycated hemoglobin A1c (HbA1c: NGSP; National Glycohemoglobin Standardization Program) and calcium $(\mathrm{Ca})$ were measured by routine techniques using an automated analyzer. The calcium concentration was adjusted using the following equation by Payne: adjusted calcium concentration $(\mathrm{mg} / \mathrm{dL})=$ measured serum calcium $(\mathrm{mg} / \mathrm{dL})+$ 
Table 2. The baseline demographic and clinical characteristics of the 71 CAD patients in the TRACP-5b quartiles

\begin{tabular}{|c|c|c|c|c|c|}
\hline & $\begin{array}{c}\mathrm{Q} 1: \leq 285 \\
(n=18)\end{array}$ & $\begin{array}{c}\text { Q2: 286-362 } \\
(n=16)\end{array}$ & $\begin{array}{l}\text { Q3: 363-482 } \\
\quad(n=20)\end{array}$ & $\begin{array}{c}\text { Q4: }>482 \\
(n=17)\end{array}$ & $P$ value \\
\hline Age (years) & $65.2 \pm 10.3$ & $64.7 \pm 8.3$ & $70.3 \pm 7.6$ & $75.8 \pm 9.0$ & 0.001 \\
\hline Systolic blood pressure $(\mathrm{mmHg})$ & $129 \pm 20$ & $136 \pm 19$ & $143 \pm 19$ & $134 \pm 19$ & 0.157 \\
\hline Heart rate (beats/min) & $70 \pm 14$ & $68 \pm 7$ & $60 \pm 8$ & $62 \pm 9$ & 0.007 \\
\hline Active smoker (\%) & $15(83.3)$ & $13(81.3)$ & $18(90.0)$ & $11(64.7)$ & 0.271 \\
\hline Hypertension (\%) & $17(94.4)$ & $16(100)$ & $18(90.0)$ & $17(100)$ & 0.364 \\
\hline Diabetes (\%) & $11(61.1)$ & $8(50.0)$ & $8(40.0)$ & $5(29.4)$ & 0.270 \\
\hline Hyperuricemia (\%) & $4(22.2)$ & $6(43.8)$ & $6(30.0)$ & $8(47.1)$ & 0.373 \\
\hline Prior PCI/CABG/Stroke/PAD (\%) & $0 / 0 / 0 / 0(0)$ & $0 / 0 / 0 / 0(0)$ & $0 / 0 / 0 / 0(0)$ & $0 / 0 / 0 / 0(0)$ & 1.000 \\
\hline Calcium channel blocker use (\%) & $6(33.3)$ & $8(50.0)$ & $8(40.0)$ & $10(45.1)$ & 0.446 \\
\hline Anti-platelet agents use (\%) & $18(100)$ & $16(100)$ & $19(95.0)$ & $17(100)$ & 0.460 \\
\hline Statin use $(\%)$ & $14(77.8)$ & $11(68.8)$ & $15(75.0)$ & $13(76.5)$ & 0.936 \\
\hline Osteoprotegerin (pmol/L) & $92.9(72.2,107.0)$ & $86.9(51.4,106.5)$ & $106.5(69.0,124.0)$ & $135.0(92.0,165.0)$ & 0.012 \\
\hline High-sensitivity C-reactive protein $(\mathrm{mg} / \mathrm{L})$ & $0.57(0.23,0.93)$ & $1.05(0.46,3.47)$ & $0.61(0.27,7.43)$ & $0.90(0.27,1.62)$ & 0.393 \\
\hline LDL cholesterol (mg/dL) & $98 \pm 16$ & $96 \pm 28$ & $89 \pm 15$ & $78 \pm 27$ & 0.035 \\
\hline HDL cholesterol (mg/dL) & $53 \pm 11$ & $57 \pm 14$ & $57 \pm 13$ & $58 \pm 15$ & 0.620 \\
\hline Triglycerides (mg/dL) & $138(114,211)$ & $92(69,108)$ & $91(75,135)$ & $114(88,163)$ & 0.523 \\
\hline Calcium (mg/dL) & $9.2 \pm 0.5$ & $9.1 \pm 0.4$ & $9.0 \pm 0.3$ & $9.3 \pm 0.7$ & 0.238 \\
\hline Albumin (g/dL) & $4.3 \pm 0.4$ & $4.1 \pm 0.4$ & $4.0 \pm 0.5$ & $4.2 \pm 0.5$ & 0.215 \\
\hline $\mathrm{eGFR}\left(\mathrm{mL} / \mathrm{min} / 1.73 \mathrm{~m}^{2}\right)$ & $59.3 \pm 13.7$ & $64.8 \pm 20.9$ & $58.3 \pm 18.6$ & $52.6 \pm 17.8$ & 0.286 \\
\hline 2 & 13 & 7 & 5 & 5 & \\
\hline 3 & 1 & 7 & 10 & 11 & \\
\hline LVEF (\%) & $58.1 \pm 11.7$ & $57.4 \pm 14.8$ & $51.0 \pm 14.9$ & $60.0 \pm 10.2$ & 0.180 \\
\hline
\end{tabular}

The data are expressed as the mean $\pm \mathrm{SD}$ or median (interquartile range). TRACP, tartrate-resistant acid phosphatase (mU/dL); differences in TRACP-5b levels among the quartiles (Q1, Q2, Q3 and Q4); PCI, percutaneous coronary intervention, CABG, coronary artery bypass grafting; PAD, peripheral artery disease; ACEI, angiotensin-converting enzyme inhibitor; LDL, low-density lipoprotein; HDL, high-density lipoprotein; eGFR, estimated glomerular filtration rate; LVEF, left ventricular ejection fraction

$(4-\text { serum albumin }[\mathrm{g} / \mathrm{dL}])^{23)}$.

\section{Statistical Analysis}

The results are presented as the mean \pm standard deviation or the median (interquartile range) for continuous variables and the percentage of the total number of patients for categorical variables. Student's $t$-test for independent samples and the chi-squared test were used to compare the continuous and categorical variables, respectively. The TRACP-5b, OPG, TG and
hsCRP levels had skewed distributions. Thus, the Mann-Whitney $U$ test was used for unpaired comparisons between the groups, and Wilcoxon's signedrank test was used for paired comparisons within the groups. The bivariate correlation between the parameters was assessed with the Pearson or Spearman correlation $(\gamma)$ coefficients for a normal or skewed distribution, respectively. The TRACP-5b levels were compared across the Gensini score quartiles and the number of coronary vessels (one-, two- and three-vessel 
Table 3. The baseline demographic and clinical characteristics of the 71 CAD patients in the Gensini score quartiles

\begin{tabular}{|c|c|c|c|c|c|}
\hline & $\begin{array}{c}\mathrm{Q} 1: \leq 35.0 \\
(n=20)\end{array}$ & $\begin{array}{c}\text { Q2: } 35.0-48.5 \\
(n=16)\end{array}$ & $\begin{array}{c}\text { Q3: 48.6-71.0 } \\
(n=17)\end{array}$ & $\begin{array}{c}\text { Q4: }>71.0 \\
(n=18)\end{array}$ & $P$ value \\
\hline Age (years) & $69.1 \pm 7.4$ & $65.5 \pm 10.3$ & $69.8 \pm 9.5$ & $71.5 \pm 11.2$ & 0.340 \\
\hline Systolic blood pressure $(\mathrm{mmHg})$ & $129 \pm 20$ & $136 \pm 19$ & $143 \pm 19$ & $134 \pm 19$ & 0.140 \\
\hline Heart rate (beats/min) & $70 \pm 14$ & $68 \pm 7$ & $60 \pm 8$ & $62 \pm 9$ & 0.190 \\
\hline Osteoprotegerin (pmol/L) & $83.1(57.0,120.8)$ & $112.0(81.0,148.0)$ & $82.5(72.8,98.2)$ & $98.3(63.6,111.0)$ & 0.403 \\
\hline High-sensitivity CRP (mg/L) & $0.57(0.21,0.97)$ & $0.63(0.34,1.48)$ & $0.76(0.28,1.81)$ & $1.62(0.47,7.61)$ & 0.163 \\
\hline LDL cholesterol (mg/dL) & $111 \pm 29$ & $124 \pm 42$ & $108 \pm 26$ & $112 \pm 34$ & 0.520 \\
\hline HDL cholesterol (mg/dL) & $53 \pm 14$ & $53 \pm 9$ & $51 \pm 10$ & $51 \pm 14$ & 0.869 \\
\hline Calcium (mg/dL) & $9.0 \pm 0.3$ & $9.1 \pm 0.4$ & $8.8 \pm 1.6$ & $8.8 \pm 2.3$ & 0.923 \\
\hline 1 & 6 & 5 & 1 & 0 & \\
\hline 2 & 12 & 8 & 7 & 3 & \\
\hline 3 & 2 & 3 & 9 & 15 & \\
\hline LVEF (\%) & $58.9 \pm 12.7$ & $57.7 \pm 13.9$ & $53.6 \pm 12.4$ & $50.5 \pm 15.2$ & 0.041 \\
\hline Hypertension (\%) & $18(90.0)$ & $15(93.8)$ & $17(100)$ & $18(100)$ & 0.340 \\
\hline Diabetes (\%) & $12(60.0)$ & $8(50.0)$ & $6(35.3)$ & $6(33.3)$ & 0.306 \\
\hline Active smoker (\%) & $17(85.0)$ & $12(75.0)$ & $12(70.6)$ & $16(88.9)$ & 0.491 \\
\hline
\end{tabular}

The data are expressed as the mean \pm SD or median (interquartile range). TRACP, tartrate-resistant acid phosphatase; differences in Gensini scores among the quartiles (Q1, Q2, Q3 and Q4); CRP, C-reactive protein; LDL, low-density lipoprotein; HDL, high-density lipoproten; eGFR, estimated glomerular filtration rate; LVEF, left ventricular ejection fraction

disease) using a two-way analysis of variance, followed by Bonferroni correction. Graphs were prepared using box-and-whisker plots with the median values and the 25 th and 75 th interquartile ranges. The circles represent outliers ( $>1.5 \%$ of the $75 \%$ confidence interval).

The association between the TRACP-5b levels and other variables was explored using a multiple linear regression analysis. The variables that were examined included the age, gender, SBP, eGFR, LDL cholesterol, HDL cholesterol, HbAlc, Ca, hsCRP, and OPG levels, as well as the use of angiotensin-converting enzyme inhibitor (ACEI), the Gensini score (model 1), or the number of diseased vessels (model 2). The Statistical Software Package for Social Science (SPSS) for Windows software program (version 18.0; SPSS, Chicago, IL, USA) was used for all of the statistical analyzes. $P$ values of $<0.05$ were considered to indicate statistical significance.

\section{Results}

The baseline demographic and clinical characteristics of the $71 \mathrm{CAD}$ patients and 28 normal healthy subjects are shown in Table 1. The LDL-cholesterol,
TG, FPG, hsCRP, and TRACP-5b levels were significantly higher in patients with $\mathrm{CAD}$ than in normal healthy subjects. The body mass index (BMI) and SBP tended to be higher in patients with CAD than in normal healthy subjects, whereas the TC levels tended to be lower in patients with CAD than in normal healthy subjects. However, there were no significant differences between the two groups in terms of the age, gender, heart rate, HDL cholesterol or eGFR values (Table 1).

The baseline demographic and clinical characteristics of the 71 CAD patients in the TRACP- 5 b quartiles are summarized in Table 2. The serum levels of TRACP-5b were divided into quartiles, as follows: first (Q1; $n=18), \leq 285 \mathrm{mU} / \mathrm{dL}$; second (Q2; $n=16), 286-$ $362 \mathrm{mU} / \mathrm{dL}$; third (Q3; $n=20), 363-482 \mathrm{mU} / \mathrm{dL}$; and fourth (Q4; $n=17),>482 \mathrm{mU} / \mathrm{dL}$.

Patients with higher TRACP-5b levels tended to be older and had higher OPG levels and Gensini scores than those with lower TRACP-5b levels, and were significantly associated with an increased number of diseased coronary vessels. Patients with higher TRACP$5 \mathrm{~b}$ levels also had lower heart rate, BMI, waist circumference and LDL cholesterol values (Table 2), and 
Table 4. The baseline demographic and clinical characteristics of the $71 \mathrm{CAD}$ patients according to the number of diseased vessels

\begin{tabular}{|c|c|c|c|c|}
\hline & $\begin{array}{l}\text { One-vessel } \\
(n=12)\end{array}$ & $\begin{array}{l}\text { Two-vessel } \\
(n=30)\end{array}$ & $\begin{array}{c}\text { Three-vessel } \\
\quad(n=29)\end{array}$ & $P$ value \\
\hline Age (years) & $71.6 \pm 7.8$ & $66.3 \pm 9.4$ & $70.8 \pm 10.2$ & 0.115 \\
\hline Systolic blood pressure ( $\mathrm{mmHg}$ ) & $129 \pm 15$ & $130 \pm 18$ & $127 \pm 21$ & 0.806 \\
\hline Heart rate (beats/min) & $67 \pm 12$ & $66 \pm 8$ & $70 \pm 8$ & 0.181 \\
\hline Osteoprotegerin (pmol/L) & $113.5(83.7,153.3)$ & $92.0(64.7,113.8)$ & $81.3(56.7,108.5)$ & 0.126 \\
\hline High-sensitivity CRP (mg/L) & $0.36(0.22,6.01)$ & $0.69(0.27,0.96)$ & $1.11(0.42,3.27)$ & 0.201 \\
\hline LDL cholesterol (mg/dL) & $104 \pm 38$ & $115 \pm 33$ & $115 \pm 31$ & 0.553 \\
\hline HDL cholesterol (mg/dL) & $58 \pm 12$ & $51 \pm 12$ & $51 \pm 12$ & 0.075 \\
\hline Calcium (mg/dL) & $9.1 \pm 0.3$ & $8.9 \pm 1.3$ & $8.8 \pm 1.7$ & 0.836 \\
\hline LVEF (\%) & $54.4 \pm 13.6$ & $56.6 \pm 13.4$ & $54.1 \pm 14.4$ & 0.767 \\
\hline Hypertension (\%) & $11(91.7)$ & $28(93.3)$ & $29(100)$ & 0.329 \\
\hline Diabetes (\%) & $9(75.0)$ & $14(46.7)$ & $9(31.0)$ & 0.035 \\
\hline Active smoker (\%) & $10(83.3)$ & $25(83.3)$ & $22(75.9)$ & 0.739 \\
\hline
\end{tabular}

The data are expressed as the mean \pm SD or median (interquartile range). TRACP, tartrate-resistant acid phosphatase; differences in the number of diseased vessels among the three groups (one-vessel, two-vessel, three-vessel); CRP, C-reactive protein; LDL, low-density lipoprotein; HDL, highdensity lipoprotein; eGFR, estimated glomerular filtration rate; LVEF, left ventricular ejection fraction

tended to have lower FPG levels than those with lower TRACP-5b levels. There were no significant differences in the other variables among the quartiles, including the prevalence of a history of myocardial infarction (Table 2). In addition, neither group included any patients with a history of PCI, CABG, stroke, or PAD prior to enrollment (Table 2).

The demographic and clinical characteristics of the CAD patients in the Gensini score quartiles are summarized in Table 3. The Gensini scores were divided into quartiles, as follows: first (Q1; $n=20), \leq 35.0$; second (Q2; $n=16), 35.0-48.5$; third (Q3; $n=17)$, 48.6-71.0; and fourth (Q4; $n=18),>71.0$. Patients with higher Gensini scores had higher TRACP-5b levels than those with lower scores and were significantly associated with an increased number of diseased coronary vessels. Patients with higher Gensini scores also had lower HbA1c and LVEF values (Table 3), and tended to have lower eGFR levels than those with lower scores. There were no significant differences in any other variables among the quartiles (Table 3).

The demographic and clinical characteristics of the patients in the three groups according to the number of diseased vessels (one-vessel, two-vessel and threevessel disease) are shown in Table 4. Patients with an increased number of diseased vessels had higher TRACP-5b and Gensini score values, and unexpect- edly had a lower prevalence of diabetes than those with fewer diseased vessels. Those with an increased number of diseased vessels also tended to have lower HDL cholesterol and HbA1c values than those with fewer diseased vessels. There were no significant differences in any other variables among the three groups (Table 4).

The relationships between the TRACP-5b level and the number of diseased vessels and the Gensini scores are shown in Fig. 1A and $\mathbf{1 B}$, respectively. Fig. 1A shows the differences in the TRACP-5b levels among the groups with one- $(n=12)$, two- $(n=30)$ and three-vessel $(n=29)$ disease. Higher TRACP-5b levels were significantly associated with an increased severity of CAD, as assessed by the number of diseased vessels $(p=0.018$, Fig. 1A). Fig. 1B shows the differences in the TRACP-5b levels among the Gensini score quartiles (Q1, Q2, Q3, and Q4). Similarly, higher TRACP-5b levels were significantly associated with an increased extent of coronary atherosclerosis, as estimated by the Gensini scores $(p=0.038)$.

To elucidate the pathophysiological mechanisms by which the TRACP-5b level predicts the severity and extent of coronary atherosclerosis, we investigated the relationships between the TRACP-5b level and other variables, including the number of diseased vessels (model 1, Table 5) and the Gensini score (model 


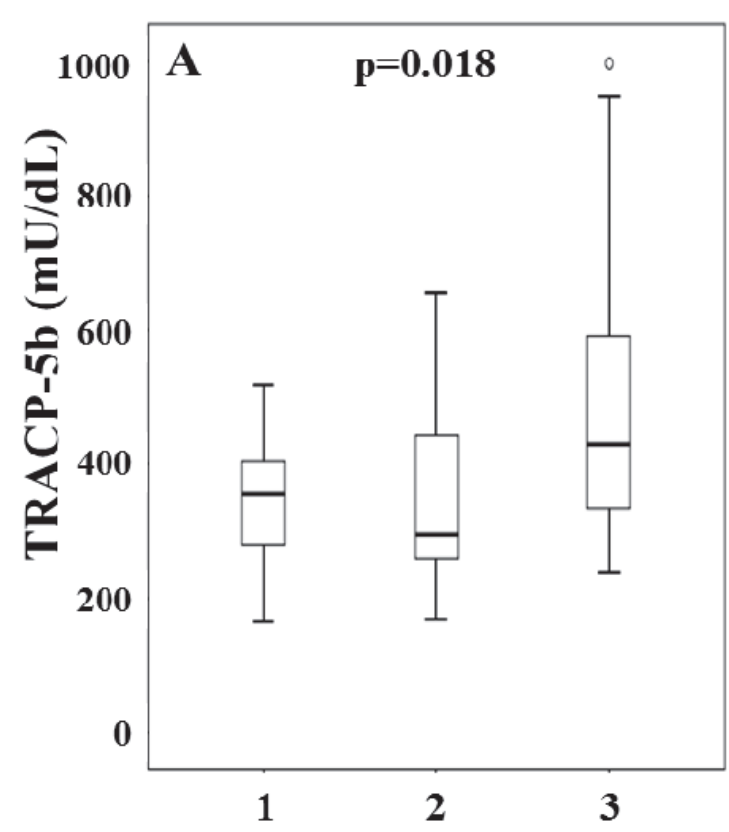

Number of diseased vessels

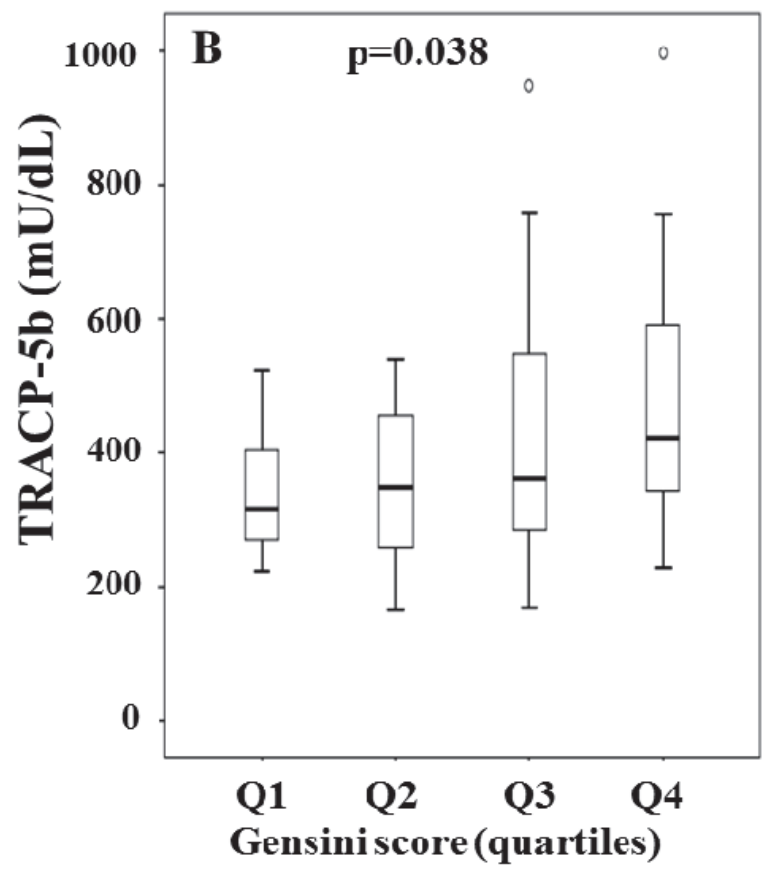

Fig. 1. Relationships between the TRACP-5b levels and the number of diseased vessels and the Gensini scores. (A) The differences in the TRACP-5b levels among the groups with one- $(n=12)$, two- $(n=30)$ and three-vessel $(n=29)$ disease. Higher TRACP-5b levels were significantly associated with an increased severity of CAD, as assessed by the number of diseased vessels $(p=0.018)$. (B) The differences in the TRACP-5b levels among the Gensini score quartiles (Q1, Q2, Q3, and Q4). Similarly, higher TRACP$5 \mathrm{~b}$ levels were significantly associated with an increased extent of coronary atherosclerosis as estimated by the Gensini score $(p=0.038)$. The box and whisker plots show the median levels and the 25 th and 75th interquartile ranges (delineated by the bottom and top of each box), and the circles represent outliers $(>1.5 \%$ of the $75 \%$ confidence interval).

2, Table 6). A multiple linear regression analysis showed that the OPG level ( $\beta$ coefficient $=0.355, p=0.003$ ), eGFR ( $\beta$ coefficient $=-0.264, p=0.021)$, and the number of diseased vessels ( $\beta$ coefficient $=0.365, p<$ 0.001 ) were significant and independent determinants of the TRACP-5b level (model 1, Table 5). Furthermore, the OPG level ( $\beta$ coefficient $=0.291, p=0.021)$, the use of ACEIs ( $\beta$ coefficient $=0.248, p=0.024$ ), and the Gensini score $(\beta$ coefficient $=0.240, p=0.036)$ were significantly and independently associated with the TRACP-5b level (model 2, Table 6).

In addition, we also investigated the relationships between the TRACP 5-level and the OPG level, patient age, and eGFR. The TRACP 5b-level was significantly and positively associated with the OPG level $(r=0.353$, $p<0.001$, Fig. 2$)$ and patient age $(r=0.428, p<0.001$, not shown in Figure). The TRACP $5 \mathrm{~b}$ level was significantly and negatively associated with the eGFR $(r=-0.256, p=0.032$, not shown in Figure).

\section{Discussion}

Atherosclerosis and calcification of the coronary arteries occur via an active process that resembles bone formation and bone remodeling ${ }^{1-6)}$. OPG is a glycoprotein and a member of the tumor necrosis factor (TNF)-related family. It is expressed by endothelial cells, vascular smooth muscle cells, and osteoblasts, and acts as a decoy receptor for the receptor activator of nuclear factor $\kappa \mathrm{B}$ (RANK) ligand (RANKL) ${ }^{2)}$. OPG can be detected in the atherosclerotic lesions of CAD patients ${ }^{24,25)}$. Elevated concentrations of circulating OPG have been significantly associated with aortic plaque as well as with the increased prevalence of, and severity of $\mathrm{CAD}^{12,26)}$. Mangan et al. ${ }^{27)}$ showed that the upregulation of OPG causes the TNF- $\alpha$ sensitization of the endothelial cells and the migration of the monocytes to the vascular intima, which are key processes in the development of vascular atherosclerosis and calcification. Thus, OPG may play important roles in the pathogenesis and development of coronary atherosclerosis and calcification in CAD patients.

Tartrate-resistant acid phosphate (TRACP) is an iron-containing glycoprotein that is highly expressed by bone-resorbing osteoclasts, inflammatory macrophages, and dendritic cells ${ }^{17)}$. Two forms of TRACP 
Table 5. A multivariate linear regression analysis of the factors associated with the serum level of TRACP-5b (model 1)

\begin{tabular}{lrr}
\hline & $\beta$ & $P$ value \\
\hline Age (years) & 0.183 & 0.147 \\
Male gender & 0.184 & 0.077 \\
Systolic blood pressure (mmHg) & 0.026 & 0.779 \\
Osteoprotegerin (pmol/L) & 0.355 & 0.003 \\
LDL cholesterol (mg/dL) & -0.029 & 0.764 \\
HDL cholesterol (mg/dL) & 0.053 & 0.614 \\
Serum calcium $(\mathrm{mg} / \mathrm{dL})$ & -0.111 & 0.310 \\
eGFR (mL/min/1.73 m $\left.{ }^{2}\right)$ & -0.264 & 0.021 \\
HbA1c $(\%)$ & -0.063 & 0.516 \\
High sensitivity CRP $(\mathrm{mg} / \mathrm{L})$ & -0.037 & 0.701 \\
ACEI use & 0.178 & 0.071 \\
Number of diseased vessels $\geq 70 \%$ & 0.365 & $<0.001$ \\
\hline
\end{tabular}

Model adjusted $\mathrm{R}^{2}=0.442$

$\beta$, regression coefficient; LDL, low-density lipoprotein; HDL, highdensity lipoprotein; eGFR, estimated glomerular filtration rate; CRP:

C-reactive protein; ACEI, angiotensin-converting enzyme inhibitor

circulate in the human blood: TRACP-5a, which is derived from macrophages and dendritic cells, and TRACP-5b, which is derived from osteoclasts ${ }^{17,18)}$. Recent studies have shown that serum TRACP-5b activity is correlated with bone resorption markers and osteoclast numbers ${ }^{17)}$. In addition, the serum TRACP$5 \mathrm{~b}$ activity is a promising new marker with clinical relevance in diseases such as RA, CKD, and bone metastasis in various cancers with increased bone resorption and destruction ${ }^{17-19)}$. Thus, both OPG and TRACP$5 \mathrm{~b}$ are sensitive and specific markers of bone resorption and bone remodeling.

However, no previous studies have investigated the relationship between the serum levels of TRACP$5 \mathrm{~b}$ and the severity or extent of coronary atherosclerosis in CAD patients. The present study provides important evidence showing that the serum levels of TRACP-5b are significantly associated with the severity of CAD (as assessed by the number of diseased coronary vessels) and the extent of coronary atherosclerosis (as indicated by the Gensini score). The patients with higher TRACP-5b levels tended to be older and had higher OPG levels and lower BMI and LDL cholesterol values than those with lower TRACP-5b levels. In addition, we also showed that the serum levels of TRACP-5b were significantly higher in patients with CAD than in normal healthy subjects. Furthermore, the patients with higher Gensini scores had higher TRACP-5b levels, as did those with an increased number of diseased vessels.

A multivariate linear regression analysis showed that the OPG levels were significantly and indepen-
Table 6. A multivariate linear regression analysis of the factors associated with the serum level of TRACP-5b (model 2)

\begin{tabular}{lcc}
\hline & $\beta$ & $P$ value \\
\hline Age (years) & 0.258 & 0.055 \\
Male gender & 0.131 & 0.246 \\
Systolic blood pressure (mmHg) & 0.065 & 0.565 \\
Osteoprotegerin (pmol/L) & 0.292 & 0.021 \\
LDL cholesterol (mg/dL) & 0.040 & 0.695 \\
HDL cholesterol (mg/dL) & 0.007 & 0.952 \\
Serum calcium $(\mathrm{mg} / \mathrm{dL})$ & -0.120 & 0.313 \\
eGFR (mL/min/1.73 m $\left.{ }^{2}\right)$ & -0.226 & 0.067 \\
HbA1c $(\%)$ & -0.077 & 0.465 \\
High sensitivity CRP (mg/L) & -0.094 & 0.364 \\
ACEI use & 0.248 & 0.024 \\
Gensini score & 0.240 & 0.036 \\
\hline
\end{tabular}

Model adjusted $\mathrm{R}^{2}=0.356$

$\beta$, regression coefficient; LDL, low-density lipoprotein; HDL, highdensity lipoprotein; eGFR, estimated glomerular filtration rate; CRP: C-reactive protein; ACEI, angiotensin-converting enzyme inhibitor

dently correlated with the serum levels of TRACP-5b. These findings suggest that the serum levels of TRACP$5 \mathrm{~b}$ are significantly associated with the plasma levels of OPG, which are a sensitive and specific marker of bone reabsorption and bone remodeling in CAD patients.

Finally, the present study demonstrated, for the first time that the serum levels of TRACP- $5 \mathrm{~b}$ are significantly and independently associated with the plasma levels of OPG and with the severity and extent of coronary atherosclerosis in CAD patients. These data provide important information for determining optimum therapeutic strategies for patients with CAD.

The mechanisms underlying the significant relationship between the serum levels of TRACP-5b and the severity and extent of coronary atherosclerosis in CAD patients are unknown. Epidemiological studies have shown that osteoporosis, which contributes to morbidity and mortality independently of age, often coexists with atherosclerosis, calcification and cardiovascular diseases, including $\mathrm{CAD}^{28-30}$.

Parham et al. ${ }^{31)}$ showed that an atherogenic highfat diet induced dyslipidemia and lipid oxidation, such as oxidized LDL cholesterol, which promoted atherosclerosis and reduced bone mineralization in mice. Tintut $e t a .^{32)}$ showed that TNF- $\alpha$ induced by a highfat diet promoted in vitro vascular calcination via the cyclic AMP pathway.

Tintut et al. ${ }^{33)}$ also showed that the osteoclastic activity as indicated by the TRACP activity was also significantly higher in LDL receptor knockout mice, which showed elevated serum cholesterol levels and 


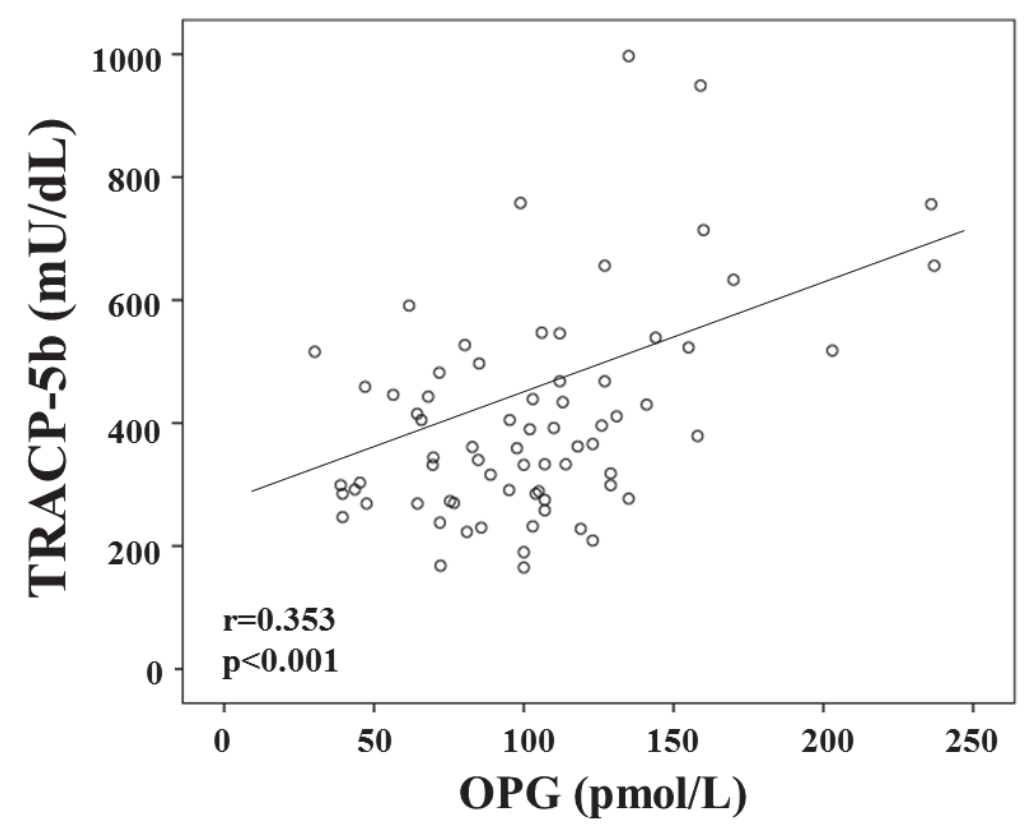

Fig. 2. Relationship between the TRACP-5b levels and the OPG levels. The TRACP-5b levels were significantly and positively associated with the OPG levels $(r=0.353, p<0.001)$.

developed atherosclerotic plaques to different extents under specific dietary conditions, than in wild-type mice. These data suggest that dyslipidemia may contribute to the pathogenesis and development of osteoporosis via an increased osteoclastic bone resorption, as estimated by the TRACP activity. However, the present study showed that lipid profiles, including LDL and HDL cholesterols were not significantly and independently associated with the TRACP-5b levels (Tables 5 and $\mathbf{6}$ ).

Increased OPG levels have been associated with the presence and severity of coronary atherosclerosis. However, contradictory evidence does exist; some investigators have shown OPG not to be associated with either calcification or the severity of coronary atherosclerosis ${ }^{34-36)}$. Despite the fact of this study that the OPG level was significantly and independently associated with the TRACP-5b level. However, the present study showed the OPG levels not to be associated with the severity or extent of coronary atherosclerosis (Tables 3 and 4). Interestingly, Poulsen et al. ${ }^{34)}$ showed that an increased OPG concentration was related to carotid and peripheral artery disease, but not to myocardial ischemia, in patients with type 2 diabetes. In addition, we have previously shown that OPG levels were significantly related to the carotid atherosclerosis estimated by carotid intima-media thickness but not associated with the severity and extent of coronary atherosclerosis in patients with

\section{$\mathrm{CAD}^{37)}$.}

OPG is a key factor in bone remodeling and is a sensitive and specific marker of bone resorption ${ }^{2}$, while the serum TRACP-5b activity is a promising new marker with clinical relevance in diseases such as RA, $\mathrm{CKD}$ and bone metastasis in various cancers that are associated with increased bone resorption and destruction ${ }^{17,19)}$. These data suggest that both TRACP-5b and OPG levels are sensitive and specific markers of bone resorption and bone remodeling in patients with CKD and RA. However, the TRACP-5b levels may be superior to the OPG levels in predicting the severity and extent of coronary atherosclerosis in patients with CAD. These discrepancies may be related to differences in the length (short) of the follow-up period, the frequent uses of modern pharmacotherapy, including statins, ACEI or angiotensin receptor blocker, the evaluation of relatively low-risk patient populations (with or without CAD), or the use of a small sample size.

Finally, we hypothesize that elevated TRACP-5b activity may be associated with the OPG levels as well as with the severity and extent of coronary atherosclerosis in CAD patients.

\section{Study Limitations}

The present study is associated with several possible limitations. The most significant limitation is the small sample size. In addition, we only studied CAD 
patients; as such, our results may not be applicable to the general population. Second, the majority of CAD patients had been treated with various medications, including statins, prior to their enrollment in the present study. Thus, all of the eligible patients in the present study were stabilized with the above medications, suggesting that the TRACP-5b and OPG levels may have already been stabilized by these medications. Third, we did not estimate the coronary calcification using computed tomography. Fourth, we only measured the serum levels of TRACP-5b at entry.

\section{Conclusion}

In conclusion, the serum levels of TRACP-5b were significantly and independently associated with the OPG levels as well as with the severity and extent of coronary atherosclerosis in CAD patients. Further studies are needed to confirm these findings.

\section{Acknowledgements}

The authors thank Ms. Sanae Otsudo for her helpful assistance in collecting the data.

\section{Disclosures}

The authors declare no conflicts of interest in association with the present study.

\section{References}

1) Alexopoulos N, Raggi P. Calcification in atherosclerosis. Nat Rev Cardiol 2009; 6: 681-688

2) Campenhout V. Golledge J. Osteoprotegerin, vascular calcification and atherosclerosis. Atherosclerosis 2009; 204: 321-329

3) Johnson RC, Leopold JA, Loscalzo J. Vascular calcification. Pathobiological mechanisms and clinical implications. Circ Res 2006; 99: 1044-1059

4) Demer LL, Tintut Y. Vascular calcification. Pathobiology of a multifaceted disease. Circulation 2008; 117: 29382948

5) New SE, Aikawa E. Cardiovascular calcification: an inflammatory disease. Circ J 2011; 75: 1305-1313

6) Hangai M, Takebe N, Honma H, Sasaki A, Chida A, Nakano R, Togashi H, Nakagawa R, Oda T, Matsui M, Yashiro S, Nagasawa K, Kajiwara T, Takahashi K, Takahashi Y, Satoh J, Ishigaki Y. Association of Advanced Glycation End Products with coronary Artery Calcification in Japanese Subjects with Type 2 Diabetes as Assessed by Skin Autofluorescence. J Atheroscler Thromb 2016; 23: 1178-1187

7) Tsuruda T. Osteoprotegerin: osteo-vascular connection. J Atheroscler Thromb 2015; 26: 233-234

8) Pal SN, Golledge J. Osteo-progenitors in vascular calcifi- cation: a circulating cell theory. J Atheroscler Thromb 2011; 18: 551-559

9) Kurnatowska I, Grzelak P, Kaczmarska M, Stefańczyk L, Nowicki M. Serum osteoprotegerin is a predictor of progression of atherosclerosis and coronary calcification in hemodialysis patients. Nephron Clin Pract 2011; 117: c297-c304

10) Svensson M, Dahle DO, Mjøen G, Weihrauch G, Scharnagl H, Dobnig H, März W, Jardine A, Fellström B, Holdaas $\mathrm{H}$. Osteoprotegerin as a predictor of renal and cardiovascular outcomes in renal transplant recipients: follow-up data from the ALERT study. Neprol Dial Transplant 2012; 27: 2571-2575

11) Cao H, Wang J, Xi L, Røe OD, Chen Y, Wang D. Dysregulated atrial gene expression of osteoprotegerin/receptor activator of nuclear factor- $\kappa \mathrm{B}$ (RANK)/RANK ligand axis in the development and progression of atrial fibrillation. Circ J 2011; 75: 2781-2788

12) Jono S, Ikari Y, Shioi A, Mori K, Miki T, Hara K, Nishizawa Y. Serum osteoprotegerin levels are associated with the presence and severity of coronary artery disease. Circulation 2002; 106: 1192-1194

13) Rhee EJ, Lee WY, Kim SY, Kim BJ, Sung KC, Kim BS, Kang JH, Oh KW, Oh ES, Baek KH, Kang MI, Woo HY, Park HS, Kim SW, Lee MH, Park JR. Relationship of serum osteoprotegerin levels with coronary artery disease severity, left ventricular hypertrophy and C-reactive protein. Clin Sci (Lond) 2005; 108: 237-243

14) Venuraju SM, Yerramasu A, Corder R, Lahiri A. Osteoprotegerin as a predictor of coronary artery disease and cardiovascular mortality and morbidity. J Am Coll Cardiol 2010; 55: 2049-2061

15) Lee CJ, Wang JH, Chen ML, Yang CF, Chen YC, Hsu BG. Serum osteoprotegerin is associated with arterial stiffness assessed according to the cardio-ankle vascular index in hypertensive patients. J Atheroscler Thromb 2015; 22: 304-312

16) Vik A, Mathiesen EB, Brox J, Wilsgaard T, Njølstad I, Jørgensen L, Hansen JB. Relation between serum osteoprotegerin and carotid intima media thickness in a general population - the Tromsø Study. J Thromb Haemost 2010; 8: 2133-2139

17) Janckila AJ, Slone SP, Lear SC, Martin A, Yam LT. Tartrate-resistant acid phosphatase as an immunohistochemical marker for inflammatory macrophages. Am J Clin Pathol 2007; 127: 556-566

18) Wu YY, Janckila AJ, Slone SP, Perng WC, Chao TY. Tartrate-resistant acid phosphatase $5 \mathrm{a}$ in sarcoidosis: further evidence for a novel macrophage biomarker in chronic inflammation. J Formos Med Assoc 2014; 113: 364-370

19) Chao TY, Wu YY, Janckila AJ. Tartrate-resistant acid phosphatase isoform $5 \mathrm{~b}$ (TRACP $5 \mathrm{~b}$ ) as a serum maker for cancer with bone metastasis. Clin Chim Acta 2010; 411: 1553-1564

20) Nakagomi A, Celermajer DS, Lumley T, Freedman SB. Angiographic severity of coronary narrowing is a surrogate marker for the extent of coronary atherosclerosis. Am J Cardiol 1996; 78: 516-519

21) Gensini GG. A more meaningful scoring system for determining the severity of coronary heart disease. Am J Cardiol 1983; 51: 606 
22) Imai E, Horio $M$, Watanabe $T$, Iseki $K$, Yamagata $K$, Hara S, Ura N, Kiyohara Y, Moriyama T, Ando Y, Fujimoto S, Konta T, Yokoyama H, Makino H, Hishida A, Matsuo S. Prevalence of chronic kidney disease in the Japanese general population. Clin Exp Nephrol 2009; 13: 621-630

23) Payne RB, Little AJ, Williams RB, Milner JR. Interpretation of serum calcium in patients with abnormal serum proteins. Br Med J 1973; 4: 643-646

24) Omland T, Ueland T, Jansson AM, Persson A, Karlsson T, Smith C, Herlitz J, Aukrust P, Hartford M, Caidahl K. Circulating osteoprotegerin levels and long-term prognosis in patients with acute coronary syndromes. J Am Coll Cardiol 2008; 51: 627-633

25) Dhore CR, Cleutjens JP, Lutgens E, Cleutjens KB, Geusens PP, Kitslaar PJ, Tordoir JH, Spronk HM, Vermeer C, Daemen MJ. Differential expression of bone matrix regulatory proteins in human atherosclerotic plaques. Arterioscler Thromb Vasc Biol 2001; 21: 1998-2003

26) Abedin M, Omland T, Ueland T, Khera A, Aukrust P, Murphy SA, Jain T, Gruntmanis U, McGuire DK, de Lemos JA. Relation of osteoprotegerin to coronary calcium and aortic plaque (from the Dallas Heart Study). Am J Cardiol 2007; 99: 513-518

27) Mangan SH, Van Campenhout A, Rush C, Golledge J. Osteoprotegerin upregulates endothelial cell adhesion molecule response to tumor necrosis factor-alpha associated with induction of angiopoietin-2. Cardiovasc Res 2007; 76: 494-505

28) Calcification of the aorta and osteoporosis. A roentgenographic study. JAMA 1972; 219: 1307-1311

29) Frye MA, Melton LJ 3rd, Bryant SC, Fitzpatrick LA, Wahner HW, Schwartz RS, Riggs BL. Osteoporosis and calcification of the aorta. Bone Miner 1992; 19: 185-194

30) Erbilen E, Yazici S, Ozhan H, Bulur S, Ordu S, Yazici M. Relationship between angiographically documented coro- nary artery disease and low bone mass in men. Circ J 2007; 71: 1095-1098

31) Parhami F, Tintut Y, Beamer WG, Gharavi N, Goodman W, Demer LL. Atherogenic high-fat diet reduces bone mineralization in mice. J Bone Miner Res 2001; 16: 182188

32) Tintut Y, Patel J, Parhami F, Demer LL. Tumor necrosis factor-alpha promotes in vitro calcification of vascular cells via the cAMP pathway. Circulation 2000; 102: 26362642

33) Tintut Y, Morony S, Demer LL. Hyperlipidemia promotes osteoclastic potential of bone marrow cells ex vivo. Arterioscler Thromb Vasc Biol 2004; 24: e6-e10

34) Poulsen MK, Nybo M, Dahl J, Hosbond S, Poulsen TS, Johansen A, Høilund-Carlsen PF, Beck-Nielsen H, Rasmussen LM, Henriksen JE, Poulsen MK. Plasma osteoprotegerin is related to carotid and peripheral arterial disease, but not to myocardial ischemia in type 2 diabetes mellitus. Cardiovasc Diabet 2011; 10: 76-84

35) Aksu F, Ozçelik F, Kunduracilar H, Barutçu A, Yel M, Umit EG, Altun A. The relation between the levels of osteoprotegerin and the degree of coronary artery disease in patients with acute coronary syndrome and stable angina pectoris. Kardiol Pol 2014; 72: 34-41

36) Hosbond SE, Diederichsen AC, Saaby L, Rasmussen LM, Lambrechtsen J, Munkholm H, Sand NP, Gerke O, Poulsen TS, Mickley H. Can osteoprotegerin be used to identify the presence and severity of coronary artery disease in different clinical settings? Atherosclerosis 2014; 36: 230-236

37) Morisawa T, Nakagomi A, Kohashi K, Kosugi M, Kusama Y, Atarashi H, Shimizu W. Osteoprotegerin is associated with endothelial function and predicts early carotid atherosclerosis in patients with coronary artery disease. Int Heart J 2015; 56: 605-612 